Homology, Homotopy and Applications, vol.7(1), 2005, pp.169-184

\title{
HOMOTOPY ACTIONS, CYCLIC MAPS AND THEIR DUALS
}

\author{
MARTIN ARKOWITZ AND GREGORY LUPTON
}

(communicated by James Stasheff)

\begin{abstract}
An action of $A$ on $X$ is a map $F: A \times X \rightarrow X$ such that $\left.F\right|_{X}=$ id $: X \rightarrow X$. The restriction $\left.F\right|_{A}: A \rightarrow X$ of an action is called a cyclic map. Special cases of these notions include group actions and the Gottlieb groups of a space, each of which has been studied extensively. We prove some general results about actions and their Eckmann-Hilton duals. For instance, we classify the actions on an $H$-space that are compatible with the $H$-structure. As a corollary, we prove that if any two actions $F$ and $F^{\prime}$ of $A$ on $X$ have cyclic maps $f$ and $f^{\prime}$ with $\Omega f=\Omega f^{\prime}$, then $\Omega F$ and $\Omega F^{\prime}$ give the same action of $\Omega A$ on $\Omega X$. We introduce a new notion of the category of a map $g$ and prove that $g$ is cocyclic if and only if the category is less than or equal to 1 . From this we conclude that if $g$ is cocyclic, then the Berstein-Ganea category of $g$ is $\leqslant 1$. We also briefly discuss the relationship between a map being cyclic and its cocategory being $\leqslant 1$.
\end{abstract}

\section{Introduction}

Group actions on spaces are natural objects of study in topology. Their analogues in homotopy theory lead to the notion of a homotopy action of one space on another. The restriction to the basepoint of $X$ of a group action $G \times X \rightarrow X$ yields a map $G \rightarrow X$, known as the orbit map of the action. These maps, and their homotopytheoretic analogues, are frequently of interest. For instance, the $n$th Gottlieb group of a space $X$, denoted $G_{n}(X)$, may be defined as homotopy classes of maps $f: S^{n} \rightarrow X$ such that $\left(f \mid\right.$ id) $: S^{n} \vee X \rightarrow X$ admits an extension $S^{n} \times X \rightarrow X$ up to homotopy [Got69]. That is, the $n$th Gottlieb group is the set of homotopy classes of orbit maps of $S^{n}$-actions on $X$. These groups can be generalized. For example, in [Var69] the homotopy set $G(A, X)$ of cyclic maps from $A$ to $X$ is defined as the homotopy classes of maps $f: A \rightarrow X$ such that $(f \mid$ id $): A \vee X \rightarrow X$ admits an extension $A \times X \rightarrow X$.

Received April 24, 2005, revised June 7, 2005; published on June 20, 2005.

2000 Mathematics Subject Classification: 55Q05, 55M30, 55P30.

Key words and phrases: Action, cyclic map, category of a map, coaction, cocyclic map, cocategory of a map.

(C) 2005, Martin Arkowitz and Gregory Lupton. Permission to copy for private use granted. 
Such an extension is called an $f$-action, and so a cyclic map $f: A \rightarrow X$ is just the orbit map of an $f$-action of $A$ on $X$. Actions and cyclic maps have been well-studied in the literature. For the former, see [Gan67a, Zab76, IO03]. For the latter, see [Var69, HV75, LW01]. The Gottlieb groups have also received much attention in rational homotopy, following the results of Félix-Halperin in [FH82].

In this paper we prove some general results about actions and cyclic maps. In Section 2, we focus on actions and the operations on homotopy sets that are induced by actions. We deduce several formulas, such as one for the homomorphism induced on homotopy sets (Lemma 2.5). We also conclude that any two actions loop to the same action (Proposition 2.8), which implies that any two multiplications on an $H$-space loop to the same multiplication (Corollary 2.9). Our results dualize, and we summarize the dual results at the end of Section 2. In Section 3, we concentrate on the dual of a cyclic map, called a cocyclic map. We introduce a new version of the Lusternik-Schnirelmann category of a map and show that the maps of category $\leqslant 1$ are precisely the cocyclic ones (Theorem 3.7). Since there has been increasing interest in Lusternik-Schnirelmann category recently (see [CLOT03]), this gives motivation for the study of cocyclic maps. Furthermore, we clarify the relation between cocyclic maps and connecting maps of cofibration sequences (Theorem 3.16). In doing so, we fill a gap in the literature. The relation between cyclic maps and connecting maps of fibration sequences has long been well-understood [Got69, HV75]. The dual situation, however, has remained unclear due to the lack of a dual for the classifying space construction.

All spaces, maps and homotopies are assumed to be based. All spaces are assumed to be CW complexes. We use $*: X \rightarrow Y$ and id: $X \rightarrow X$ to denote, respectively, the constant map and the identity map. We denote the set of homotopy classes of maps from $X$ to $Y$ by $[X, Y]$. However, we often do not distinguish between a map and the homotopy class it represents. In Section 3 we frequently consider lifts and sections of certain maps, by which we mean lifts and sections up to homotopy. If $f: A \rightarrow B$ is a map, then $f^{*}$ denotes pre-composition by $f$ and $f_{*}$ denotes postcomposition by $f$. Thus we obtain maps of homotopy sets $f^{*}:[B, X] \rightarrow[A, X]$ and $f_{*}:[X, A] \rightarrow[X, B]$. We also use $f^{*}$ to denote the map induced by $f$ on cohomology and $f_{\#}$ to denote the map induced on homotopy groups. The wedge of spaces $X$ and $Y$ is denoted $X \vee Y$. The unit interval $[0,1]$ is denoted $I$. The cone on a space $X$ is written $C X$ and the path space is $E X$. The loop space, respectively suspension, of a space $X$ is denoted $\Omega X$, respectively $\Sigma X$. We recall that the standard multiplication $\mu$ on $\Omega Y$ induces a group structure in $[X, \Omega Y]$ and the standard comultiplication $\nu$ on $\Sigma X$ induces a group structure in $[\Sigma X, Y]$. These group structures are isomorphic via adjointness, that is, $[X, \Omega Y] \cong[\Sigma X, Y]$. Under this isomorphism, the identity id: $\Omega X \rightarrow \Omega X$ has an adjoint which we denote $p: \Sigma \Omega X \rightarrow X$ and the identity id: $\Sigma X \rightarrow \Sigma X$ has an adjoint which we denote by $e: X \rightarrow \Omega \Sigma X$. By an $H$-space, we mean a pair $(X, m)$ with $m: X \times X \rightarrow X$ a (not-necessarily associative) multiplication up to homotopy. Dually, by a co-H space we mean a pair $(X, n)$ with $n: X \rightarrow X \vee X$ a (not-necessarily associative) comultiplication up to homotopy. 


\section{Actions on Spaces and Operations on Homotopy Sets}

In this section we consider the action of one space on another, and cyclic maps from one space to another. We present several general results about these notions that illustrate the relation between them. At the end of the section, we briefly consider the dual notions of coactions and cocyclic maps.

Let $i_{1}: A \rightarrow A \times X$ and $i_{2}: X \rightarrow A \times X$ denote the inclusions.

Definition 2.1. By a (left) action of $A$ on $X$, we mean a map $F: A \times X \rightarrow X$ that satisfies $F \circ i_{2}=$ id: $X \rightarrow X$. If $F \circ i_{1}=f: A \rightarrow X$, then we refer to $F$ as an $f$-action. Given an $f$-action $F$, we say that $f$ is the orbit map of $F$. A map $f: A \rightarrow X$ is called a cyclic map if it is the orbit map of some $f$-action $F$. We denote the set of cyclic homotopy classes $G(A, X) \subseteq[A, X]$.

In the literature, an $f$-action is often referred to as an affiliated map (of $f$ ). There is an alternate point of view on actions and cyclic maps that is often convenient to adopt: Let $X$ be a space with basepoint $x_{0}$ and $\operatorname{map}(X, X$;id $)$ denote the component of the identity in the function space of unbased maps from $X$ to itself. Let $\omega: \operatorname{map}(X, X ;$ id $) \rightarrow X$ denote the evaluation map defined by $\omega(h)=h\left(x_{0}\right)$, where $x_{0}$ is the base point of $X$. Then an $f$-action $F: A \times X \rightarrow X$ corresponds to a lift $\tilde{f}: A \rightarrow \operatorname{map}(X, X$; id) of $f$ through $\omega$, that is, $\omega \circ \tilde{f}=f$. This correspondence is given by $\tilde{f}(a)(x)=F(a, x)$. Then the set of cyclic maps $G(A, X)$ may be described as the image of the induced map of homotopy sets $\omega_{*}:[A, \operatorname{map}(X, X ;$ id $)] \rightarrow[A, X]$. It follows easily from this point of view that, if $A$ is a suspension, then $G(A, X)$ is a subgroup of $[A, X][\mathbf{V a r 6 9}]$.

We illustrate these notions with some well-known examples.

Examples 2.2. 1. The projection $p_{2}: A \times X \rightarrow X$ is a $*$-action.

2. Let $(X, m)$ be an $H$-space. Then the multiplication $m: X \times X \rightarrow X$ is a id-action.

3. Suppose given an $f$-action $F: A \times X \rightarrow X$ and any map $h: A^{\prime} \rightarrow A$. Then $F \circ(h \times$ id $): A^{\prime} \times X \rightarrow X$ is an $(f \circ h)$-action. It follows that $h^{*}: G(A, X) \rightarrow$ $G\left(A^{\prime}, X\right)$. By combining this and the previous observations, we obtain: If $(X, m)$ is an $H$-space, then $m \circ(f \times$ id $): A \times X \rightarrow X$ is an $f$-action for any $f: A \rightarrow X$, and so $G(A, X)=[A, X]$.

4. Let $\omega: \operatorname{map}(X, X$;id $) \rightarrow X$ be the evaluation map as above. Define the map $W: \operatorname{map}(X, X ;$ id $) \times X \rightarrow X$ by $W(f, x)=f(x)$. Then $W$ is a (right) $\omega$-action of $\operatorname{map}(X, X ;$ id $)$ on $X$. Indeed, $W$ is a "universal action" on $X$, in that every action of a space $A$ on $X$ factors through $W$. From this we see that $\omega$ is a "universal cyclic map," in the sense that it is a cyclic map through which every cyclic map to $X$ factors.

5. Suppose $A \stackrel{j}{\longrightarrow} X \stackrel{p}{\longrightarrow} B$ is a fibration. We call this a homotopy principal fibration if there exists a $j$-action $F: A \times X \rightarrow X$ of the fibre on the total 
space, such that the following diagram homotopy commutes:

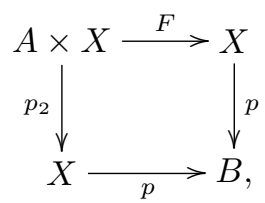

see $[\mathbf{P T 5 8}, \mathbf{P o r} 71]$. This is the obvious homotopy-theoretic analogue of the situation in which $A=G$ is a topological group and $p: X \rightarrow B$ is a principal $G$-bundle. An important special case of a homotopy principal fibration is that of a fibration $\Omega B \rightarrow E \rightarrow X$ induced from the path-space fibration over $B$ by some map $g: X \rightarrow B$.

An action of one space on another induces an operation on homotopy sets as follows. Suppose $F: A \times X \rightarrow X$ is an $f$-action and $W$ is any space. Given $a \in[W, A]$ and $h \in[W, X]$, we define $a \cdot h \in[W, X]$ as the composition

$$
W \stackrel{\Delta}{\longrightarrow} W \times W \stackrel{a \times h}{\longrightarrow} A \times X \stackrel{F}{\longrightarrow} X,
$$

where $\Delta$ is the diagonal map (see [Hil65, Ch.15] and [Zab76, p.56]). This gives an operation of the set $[W, A]$ on the set $[W, X]$ with the following properties:

(i) $* \cdot h=h$;

(ii) $a \cdot *=f_{*}(a)$;

(iii) $l^{*}(a \cdot h)=l^{*}(a) \cdot l^{*}(h)$ for any map $l: W^{\prime} \rightarrow W$;

(iv) $F=p_{1} \cdot p_{2}$ for the projections $p_{1}: A \times X \rightarrow A$ and $p_{2}: A \times X \rightarrow X$.

We now adopt the following notational convention. If $(X, m)$ is an $H$-space, we write the binary operation induced on $[W, X]$ by " ${ }_{m}$ ". On the other hand, if $(W, n)$ is a co- $H$-space, we write the binary operation induced on $[W, X]$ by " $\oplus_{n}$ ". This is defined as follows: if $a, b \in[W, X]$, then $a \oplus_{n} b=(a \mid b) \circ n \in[W, X]$, where $a \mid b \in[W \vee W, X]$ is the unique homotopy class determined by $a$ and $b$.

Example 2.3. Let $(X, m)$ be an $H$-space. If $f: A \rightarrow X$ is any map then, as in (3) of Examples 2.2, $m \circ(f \times$ id $)$ is an $f$-action. We denote this action by

$$
A c_{m}^{f}=m \circ(f \times \mathrm{id}): A \times X \rightarrow X .
$$

The induced operation of $[W, A]$ on $[W, X]$ satisfies

$$
a \cdot h=f_{*}(a)+_{m} h
$$

for all $a \in[W, A]$ and $h \in[W, X]$.

We now return to a general $f$-action $F: A \times X \rightarrow X$ and identify the operation when $W$ is a co- $H$-space.

Lemma 2.4. Suppose $(W, n)$ is a co-H-space and $F: A \times X \rightarrow X$ is an $f$-action. Then we have

$$
a \cdot h=f_{*}(a) \oplus_{n} h,
$$

for all $a \in[W, A]$ and $h \in[W, X]$. 
Proof. Observe that the diagram

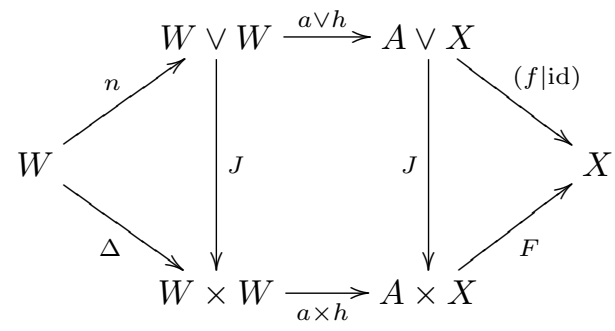

homotopy commutes, where $J$ is the inclusion.

Lemma 2.5. Let $F: A \times X \rightarrow X$ be an $f$-action and suppose given $a \in[W, A]$ and $h \in[W, X]$. Let $(U, n)$ be a co-H-space. Then the induced homomorphism $(a$. $h)_{*}:[U, W] \rightarrow[U, X]$ satisfies

$$
(a \cdot h)_{*}(\gamma)=f_{*} a_{*}(\gamma) \oplus_{n} h_{*}(\gamma),
$$

for all $\gamma \in[U, W]$.

Proof. Apply property (iii) above to $(a \cdot h)_{*}(\gamma)=\gamma^{*}(a \cdot h)$, then use Lemma 2.4.

We may specialize Lemma 2.5 to obtain a formula for the homomorphism induced on homotopy groups by a map of the form $a \cdot h$, special cases of which are used in both [LO96] and [ALM01].

Definition 2.6. Let $(X, m)$ be an $H$-space and $F: A \times X \rightarrow X$ an $f$-action. We say that the action $F$ is $m$-associative if the following diagram homotopy commutes:

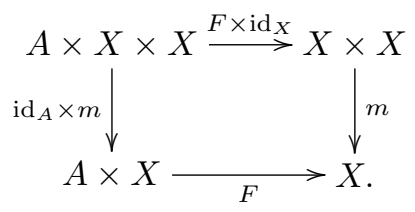

Notice that in the case in which $A=X$ and the action $F$ is the multiplication $m$, this definition reduces to that of homotopy-associativity of $m$.

Proposition 2.7. Let $(X, m)$ be an $H$-space and $f: A \rightarrow X$ a map.

1. If $m$ is homotopy-associative, then $A c_{m}^{f}$ is an $m$-associative $f$-action.

2. If $F: A \times X \rightarrow X$ is any $m$-associative $f$-action, then $F=A c_{m}^{f}$.

Proof. The first part is clear. For the second, let $i_{1}: A \rightarrow A \times X$ denote the inclusion into the first factor and $j: X \rightarrow X \times X$ the inclusion into the second factor, so that $\operatorname{id}_{A} \times j=i_{1} \times \operatorname{id}_{X}: A \times X \rightarrow A \times X \times X$. Then we have

$$
\begin{aligned}
F & =F \circ\left(\operatorname{id}_{A} \times m\right) \circ\left(\operatorname{id}_{A} \times j\right)=m \circ\left(F \times \operatorname{id}_{X}\right) \circ\left(\operatorname{id}_{A} \times j\right) \\
& =m \circ\left(\left(F \circ i_{1}\right) \times \operatorname{id}_{X}\right)=m \circ\left(f \times \operatorname{id}_{X}\right)=A c_{m}^{f} . \quad \square
\end{aligned}
$$


The following result implies that two $f$-actions loop to the same $\Omega f$-action. A similar result in a different context appears in [AOS05]. Let $\lambda: \Omega A \times \Omega X \rightarrow \Omega(A \times$ $X)$ denote the standard homeomorphism given by $\lambda(a, b)(t)=(a(t), b(t))$, where $t \in I$.

Proposition 2.8. Let $F: A \times X \rightarrow X$ be an $f$-action and let $\mu$ be the loop multiplication on $\Omega X$. Then

$$
(\Omega F) \circ \lambda=A c_{\mu}^{\Omega f}: \Omega A \times \Omega X \rightarrow \Omega X,
$$

and thus $(\Omega F) \circ \lambda$ is a $\mu$-associative $\Omega f$-action. If, in addition, $F^{\prime}: A \times X \rightarrow X$ is an $f^{\prime}$-action such that $\Omega f=\Omega f^{\prime}: \Omega A \rightarrow \Omega X$, then $\Omega F=\Omega F^{\prime}$.

Proof. Let $p: \Sigma \Omega(A \times X) \rightarrow A \times X$ be the canonical map. Then, using (iii) and (iv) of the properties listed above, we have

$$
F \circ p=p^{*}\left(p_{1} \cdot p_{2}\right)=p^{*}\left(p_{1}\right) \cdot p^{*}\left(p_{2}\right),
$$

where the last term uses the operation of $[\Sigma \Omega(A \times X), A]$ on $[\Sigma \Omega(A \times X), X]$. By Lemma 2.4, it follows that

$$
F \circ p=\left(f \circ p_{1} \circ p\right) \oplus\left(p_{2} \circ p\right),
$$

where $\oplus$ in $[\Sigma \Omega(A \times X), X]$ is induced by the suspension structure on $\Sigma \Omega(A \times X)$. By taking adjoints and noting that the adjoint of $p$ is id: $\Omega(A \times X) \rightarrow \Omega(A \times X)$, we have

$$
\Omega F=\left((\Omega f) \circ\left(\Omega p_{1}\right)\right)+{ }_{\mu} \Omega p_{2} .
$$

Let $\pi_{1}: \Omega A \times \Omega X \rightarrow \Omega A$ and $\pi_{2}: \Omega A \times \Omega X \rightarrow \Omega X$ be the projections. Then

$$
\begin{aligned}
(\Omega F) \circ \lambda & =\left((\Omega f) \circ\left(\Omega p_{1}\right) \circ \lambda\right)+{ }_{\mu}\left(\left(\Omega p_{2}\right) \circ \lambda\right) \\
& =\left((\Omega f) \circ \pi_{1}\right)+{ }_{\mu} \pi_{2}=A c_{\mu}^{\Omega f} .
\end{aligned}
$$

The other assertions of Proposition 2.8 follow easily.

The following corollary may be known, but we have not found a proof of it in the literature. It effectively answers Problem 35 from Stasheff's $H$-space problem list in [Sig71].

Corollary 2.9. If $m, m^{\prime}$ are two multiplications on $X$, then $(\Omega m) \circ \lambda=\mu=$ $\left(\Omega m^{\prime}\right) \circ \lambda$. Consequently, $\Omega m=\Omega m^{\prime}$.

For the remainder of this section, we consider the dual of the preceding discussion. Since most of this can be obtained mutatis mutandis from the previous material, we provide few details. Let $p_{1}: X \vee B \rightarrow X$ and $p_{2}: X \vee B \rightarrow B$ denote the projections.

Definition 2.10. By a (right) coaction of $B$ on $X$, we mean a map $G: X \rightarrow X \vee B$ that satisfies $p_{1} \circ G=\mathrm{id}: X \rightarrow X$. If $p_{2} \circ G=g: X \rightarrow B$, then we refer to $G$ as a $g$-coaction. Given a $g$-coaction $G$, we say that $g$ is the co-orbit map of $G$. A map $g: X \rightarrow B$ is called a cocyclic map if it is the co-orbit map of some $g$-coaction $G$. We denote the set of cocyclic homotopy classes by $G^{\prime}(X, B) \subseteq[X, B]$. 
Notice that there is no natural candidate for the dual of the function space $\operatorname{map}(X, X ;$ id $)$. So in the coaction setting, we do not have the alternative point of view that a "coevaluation map" would provide. For results on cocyclic maps, see [Var69, HV75, Lim87, LW01]. In the case in which $B=K(\pi, n)$, the set of cocyclic maps $G^{\prime}(X, B)$ has been called the $n$th dual Gottlieb group of $X$ (cf. [FLT94]).

We can dualize most of Examples 2.2; we mention two of these explicitly.

Examples 2.11. 1. Suppose given a $g$-coaction $G: X \rightarrow X \vee B$ and any map $h: B \rightarrow B^{\prime}$. Then $(\mathrm{id} \vee h) \circ G: X \rightarrow X \vee B^{\prime}$ is an $(h \circ g)$-coaction. It follows that $h_{*}: G^{\prime}(X, B) \rightarrow G^{\prime}\left(X, B^{\prime}\right)$. If $(X, n)$ is a co- $H$-space, then (id $\left.\vee g\right) \circ n: X \rightarrow$ $X \vee B$ is a $g$-coaction for any $g$, so $G^{\prime}(X, B)=[X, B]$.

2. Suppose $A \stackrel{j}{\longrightarrow} X \stackrel{p}{\longrightarrow} B$ is a cofibration. We call this a homotopy principal cofibration if there exists a $p$-coaction $G: X \rightarrow X \vee B$ such that the following diagram homotopy commutes:

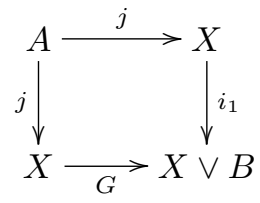

An important case occurs when the cofibration $A \rightarrow X \rightarrow \Sigma Z$ is induced from the cofibration $Z \rightarrow C Z \rightarrow \Sigma Z$ via a map $Z \rightarrow A$ [Hil65, Ch.15].

Suppose $G: X \rightarrow X \vee B$ is a coaction and $W$ is any space. Given $a \in[B, W]$ and $h \in[X, W]$, we define $h \cdot a \in[X, W]$ as the composition

$$
X \stackrel{G}{\longrightarrow} X \vee B \stackrel{h \vee a}{\longrightarrow} W \vee W \stackrel{\nabla}{\longrightarrow} W .
$$

This gives a (right) operation of the set $[B, W]$ on the set $[X, W][$ Hil65, Ch.15]. We use the same notation for this operation and the previous one. We note that properties (i)-(iv) stated earlier may be dualized.

Example 2.12. Let $(X, n)$ be a co- $H$-space. If $g: X \rightarrow B$ is any map, then (id $\vee g$ ) $\circ n$ is a $g$-coaction which we denote by $\operatorname{Coac}_{g}^{n}: X \rightarrow X \vee B$. In this case, the induced operation of $[B, W]$ on $[X, W]$ satisfies $h \cdot a=h \oplus_{n} g^{*}(a)$, for all $a \in[B, W]$ and $h \in[X, W]$.

Lemma 2.4 dualizes in a straightforward way to obtain the following.

Lemma 2.13. Suppose $(W, m)$ is an $H$-space and $G: X \rightarrow X \vee B$ is a g-coaction. Then we have

$$
h \cdot a=h+_{m} g^{*}(a),
$$

for all $a \in[B, W]$ and $h \in[X, W]$.

Definition 2.14. Let $(X, n)$ be a co- $H$-space and $G: X \rightarrow X \vee B$ a $g$-coaction. We 
say that $G$ is $n$-coassociative if the following diagram homotopy commutes:

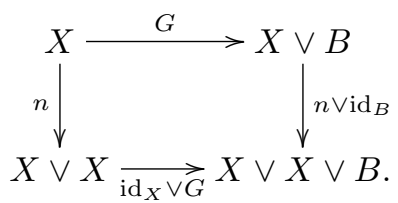

We remark that Proposition 2.7 dualizes in a straightforward way; we omit the dual statement and its proof. Let $\lambda^{\prime}: \Sigma(X \vee B) \rightarrow \Sigma X \vee \Sigma B$ denote the standard homeomorphism.

Proposition 2.15. If $G: X \rightarrow X \vee B$ is a g-coaction and $\nu$ is the suspension comultiplication on $\Sigma X$, then

$$
\lambda^{\prime} \circ(\Sigma G)=\operatorname{Coac}_{\Sigma g}^{\nu}: \Sigma X \rightarrow \Sigma X \vee \Sigma B
$$

and thus $\lambda^{\prime} \circ(\Sigma G)$ is a $\nu$-coassociative $\Sigma g$-coaction. In addition, if $G^{\prime}: X \rightarrow X \vee B$ is a $g^{\prime}$-coaction such that $\Sigma g=\Sigma g^{\prime}$, then $\Sigma G=\Sigma G^{\prime}$.

Corollary 2.16. If $n, n^{\prime}$ are two comultiplications on $X$, then $\lambda^{\prime} \circ(\Sigma n)=\nu=$ $\lambda^{\prime} \circ\left(\Sigma n^{\prime}\right)$, and so $\Sigma n=\Sigma n^{\prime}$.

We may combine the dual notions discussed above in the following interesting way. Suppose given an $f$-action $F: A \times X \rightarrow X$ and a $g$-coaction $G: X \rightarrow X \vee A$ (so $A=B$ here). These give operations of $[X, A]$ and $[A, X]$ on $[X, X]$. In particular, given any $f \in[A, X], g \in[X, A]$ and $h \in[X, X]$, we have $h \cdot f, g \cdot h \in[X, X]$ obtained using the coaction and the action, respectively.

Lemma 2.17. With the above notation, $h \cdot f=g \cdot h$. This map induces $(g \cdot h)_{\#}=$ $f_{\#} g_{\#}+h_{\#}$ on homotopy groups and $(h \cdot f)^{*}=h^{*}+g^{*} f^{*}$ on cohomology groups.

Proof. Let (id $\mid f): X \vee A \rightarrow X$ be determined by id and $f$, let (id, $g$ ) : $X \rightarrow X \times A$ be determined by id and $g$ and let $T: X \times A \rightarrow A \times X$ interchange factors. Consider the homotopy-commutative diagram

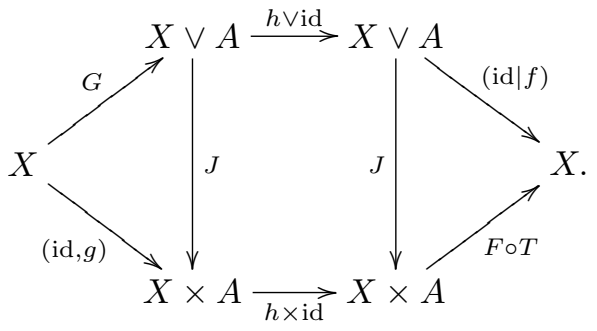

Then

$$
\begin{aligned}
h \cdot f & =(\mathrm{id} \mid f) \circ(h \vee \mathrm{id}) \circ G \\
& =F \circ T \circ(h \times \mathrm{id}) \circ(\mathrm{id}, g)=F \circ(g, h)=g \cdot h .
\end{aligned}
$$

The other assertions of Lemma 2.17 follow from Lemma 2.5 and its dual.

A special case of this situation is considered in [ALM01]. 


\section{Lusternik-Schnirelmann Category and Coactions}

In this section we relate cocyclic maps to a variant of the Lusternik-Schnirelmann category of a map. We begin by reviewing the Ganea fiber-cofiber construction [Gan67b]. In this section, we generally do distinguish between a map and its homotopy class.

Definition 3.1. Suppose given a fibration $F \stackrel{i}{\longrightarrow} E \stackrel{p}{\longrightarrow} B$. We define a sequence of fibrations

$$
F_{n}(p) \stackrel{i_{n}}{\longrightarrow} E_{n}(p) \stackrel{p_{n}}{\longrightarrow} B
$$

inductively for $n \geqslant 0$. Set $F_{0}(p) \stackrel{i_{0}}{\longrightarrow} E_{0}(p) \stackrel{p_{0}}{\longrightarrow} B$ equal to the given fibration. As-

sume $F_{n-1}(p) \stackrel{i_{n-1}}{\longrightarrow} E_{n-1}(p) \stackrel{p_{n-1}}{\longrightarrow} B$ has been defined. Set $E_{n-1}^{\prime}(p)=E_{n-1}(p) \cup_{i_{n-1}}$ $C F_{n-1}(p)$, the mapping cone of $i_{n-1}$. Define $p_{n}^{\prime}: E_{n}^{\prime}(p) \rightarrow B$ by $\left.p_{n}^{\prime}\right|_{E_{n-1}(p)}=p_{n-1}$ and $\left.p_{n}^{\prime}\right|_{C F_{n-1}(p)}=*$. Then replace $p_{n}^{\prime}$ by a fiber map to obtain a fiber sequence $F_{n}(p) \stackrel{i_{n}}{\longrightarrow} E_{n}(p) \stackrel{p_{n}}{\longrightarrow} B$.

Note that there is a map $j_{n-1}: E_{n-1}(p) \rightarrow E_{n}(p)$ such that the diagram

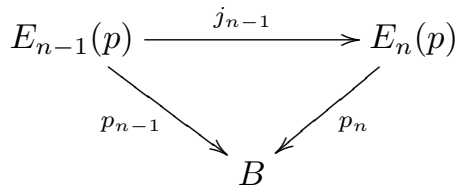

commutes.

There are two special cases of this construction that we shall need.

Examples 3.2. 1. Given a space $B$, consider the path space fibration $\Omega B \rightarrow$ $E B \rightarrow B$. Applying the above Ganea construction to this fibration, we obtain a sequence of fibrations which we write as $F_{n}(B) \stackrel{i_{n}}{\longrightarrow} G_{n}(B) \stackrel{p_{n}}{\longrightarrow} B$.

2. Given a map $g: X \rightarrow B$, we first form the fibration $\Omega B \rightarrow E \rightarrow X$ induced via $g$ from the path space fibration over $B$ by the pullback construction. Then we apply the Ganea construction to this fibration to obtain a sequence of fibrations which we write as $K_{n}(g) \stackrel{l_{n}}{\longrightarrow} H_{n}(g) \stackrel{r_{n}}{\longrightarrow} X$.

Notice that the two examples above are related. From the pullback construction, there is a map $\tilde{g}: E \rightarrow E B$ such that the following diagram commutes

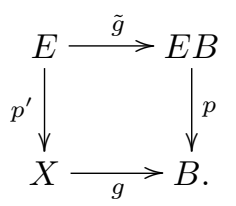

Since the Ganea construction is functorial, we get a map $\tilde{g_{n}}: H_{n}(g) \rightarrow G_{n}(B)$ such 
that the following diagram is commutative

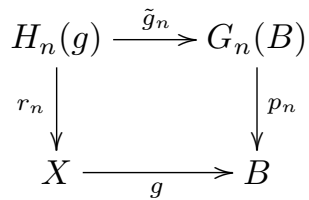

for each $n \geqslant 0$.

The constructions in Examples 3.2 lead to the following definitions of the category of a map.

Definition 3.3. Suppose given a map $g: X \rightarrow B$.

1. We say that $\operatorname{cat}_{\mathrm{BG}}(g) \leqslant n$ if $g$ can be lifted through $p_{n}$ to $G_{n}(B)$. That is, if there exists $\hat{g}_{n}: X \rightarrow G_{n}(B)$ such that

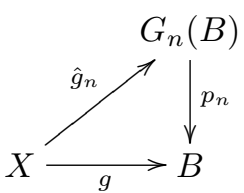

commutes up to homotopy.

2. We say that cat $(g) \leqslant n$ if $r_{n}: H_{n}(g) \rightarrow X$ has a section. That is, if there exists $s_{n}: X \rightarrow H_{n}(g)$ such that $r_{n} \circ s_{n} \simeq$ id $: X \rightarrow X$.

We define $\operatorname{cat}_{\mathrm{BG}}(g)$ (respectively, cat $\left.(g)\right)$ to be the least $n$ such that $\operatorname{cat}_{\mathrm{BG}}(g) \leqslant n$ (respectively, $\operatorname{cat}(g) \leqslant n)$.

Remarks 3.4. (1) cat $_{\mathrm{BG}}$ of a map is just the Berstein-Ganea category of a map as defined in [BG62]. However, the notion introduced in part (2) of Definition 3.3 appears to be new.

(2) We note that $\operatorname{cat}_{\mathrm{BG}}(*)=\operatorname{cat}(*)=0$, where $*: X \rightarrow Y$ is the constant map, and $\operatorname{cat}_{\mathrm{BG}}\left(\mathrm{id}_{B}\right)=\operatorname{cat}\left(\mathrm{id}_{B}\right)=\operatorname{cat}(B)$, where $\operatorname{cat}(B)$ denotes the ordinary Lusternik-Schnirelmann category of the space $B$.

(3) It follows from the definitions that $\operatorname{cat}_{\mathrm{BG}}(g) \leqslant \operatorname{cat}(g)$. We will see later that they are generally different.

Our main result in this section is that a map $g$ is cocyclic if and only if it satisfies $\operatorname{cat}(g) \leqslant 1$. To prove this, we need some notation and a preliminary result.

Notation 3.5. Given a map $g: X \rightarrow B$, define the graph of $g$ to be $\Gamma=\{(x, g(x)) \mid$ $x \in X\} \subseteq X \times B$. Let $R_{1}(g)$ denote the space $E(X \times B ; \Gamma, X \vee B)$ of paths in $X \times B$ that start in $\Gamma$ and end in $X \vee B$ with fiber map $\tilde{\pi}_{0}: R_{1}(g) \rightarrow X$ defined by $\tilde{\pi}_{0}(l)=$ the first coordinate of $l(0)$. One then obtains $R_{1}(g)$ as the fiber space over $X$ induced via (id, $g): X \rightarrow X \times B$ from the fibration over $X \times B$ obtained by converting the inclusion $J: X \vee B \rightarrow X \times B$ into a fibration. Finally, if $\gamma$ is a path in a space $W$ and $s \in I$, then $\gamma_{s}$ denotes the path in $W$ defined by $\gamma_{s}(t)=\gamma(s t)$. 
Lemma 3.6. Suppose given a map $g: X \rightarrow B$. Then there is a map $w: H_{1}(g) \rightarrow$ $R_{1}(g)$ which induces an isomorphism on homology and is such that the diagram

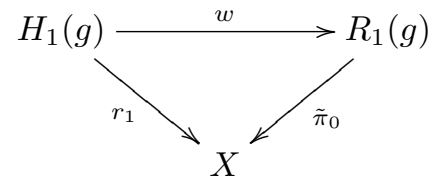

commutes.

Proof. The proof, which we sketch here, is an adaptation of an argument given by Gilbert in [Gil, Prop.3.3]. As stated earlier, $p^{\prime}: E \rightarrow X$ is induced via $g$, and so we have $\tilde{g}: E \rightarrow E B$ (see the discussion after Examples 3.2). Let $S=\{(e, \nu) \mid$ $\left.e \in E, \nu \in X^{I}, p^{\prime}(e)=\nu(0)\right\}$ and choose a lifting function $\lambda: S \rightarrow E^{I}$ for $p^{\prime}$. The extension $p_{1}^{\prime}: E \cup C \Omega B \rightarrow X$ of $p^{\prime}$ is given by $\left.p_{1}^{\prime}\right|_{E}=p^{\prime}$ and $\left.p_{1}^{\prime}\right|_{C \Omega B}=*$. Then we replace $p_{1}^{\prime}$ with a fiber map $r_{1}: H_{1}(g) \rightarrow X$, where $H_{1}(g)=\{(z, \nu) \mid z \in E \cup$ $\left.C \Omega B, \nu \in X^{I}, p_{1}^{\prime}(z)=\nu(1)\right\}$ and $r_{1}(z, \nu)=\nu(0)$. We now define $w: H_{1}(g) \rightarrow R_{1}(g)$ by

$$
w((e, s), \nu)=\left(\nu,(\tilde{g}(\lambda(e,-\nu)(1)))_{s}\right),
$$

for $s \in I,(e, s) \in E \cup C \Omega B \subseteq C E$ and $\nu \in X^{I}$ with $p_{1}^{\prime}(e, s)=\nu(1)$. One easily checks that $w$ is well-defined, has codomain $R_{1}(g)$, and satisfies $\tilde{\pi}_{0} \circ w=r_{1}$. To prove that $w$ is a homology isomorphism, it suffices to show that $\hat{w}$, the map induced on fibers, is a homology isomorphism. This is done by factoring $\hat{w}$ through $(\Omega X \times$ $C \Omega B) \cup(E X \times \Omega B) \subseteq E X \times C \Omega B$ and showing, as in the argument of Gilbert, that each of the two resulting maps induces a homology isomorphism. We omit the details.

Theorem 3.7. Let $g: X \rightarrow B$ be a map of simply connected spaces. Then $g$ is cocyclic if and only if cat $(g) \leqslant 1$.

Proof. By definition, $g$ is cocyclic if and only if (id, $g$ ): $X \rightarrow X \times B$ factors through $X \vee B$. One sees easily that this is so if and only if the fibration $\tilde{\pi}_{0}: R_{1}(g) \rightarrow X$ has a section. Since $X$ and $B$ are both simply connected, $w: H_{1}(g) \rightarrow R_{1}(g)$ is a homotopy equivalence by Lemma 3.6. It follows that $g$ is cocyclic if and only if $r_{1}: H_{1}(g) \rightarrow X$ has a section.

In the case $X=B$ and $g=\mathrm{id}$, Theorem 3.7 reduces to the following well-known result: $X$ is a co- $H$-space if and only if $p: \Sigma \Omega X \rightarrow X$ admits a section [Gan70].

The next corollary is a consequence of Remarks 3.4 (3).

Corollary 3.8. If $g: X \rightarrow B$ is a cocyclic map of simply connected spaces, then $\operatorname{cat}_{\mathrm{BG}}(g) \leqslant 1$.

We next give an example to illustrate that the converse of Corollary 3.8 does not hold.

Example 3.9. We show that the projection $p_{1}: S^{m} \times S^{n} \rightarrow S^{m}$ has $\operatorname{cat}_{\mathrm{BG}}\left(p_{1}\right) \leqslant 1$ and yet is not cocyclic. That is, we have $1=\operatorname{cat}_{\mathrm{BG}}\left(p_{1}\right)<\operatorname{cat}\left(p_{1}\right)$. Since $S^{m}$ is 
a suspension, there is a section of $p: \Sigma \Omega S^{m} \rightarrow S^{m}$ and hence a section of the first Ganea fibration $G_{1}\left(S^{m}\right) \rightarrow S^{m}$. Thus we have $\operatorname{cat}_{\mathrm{BG}}\left(p_{1}\right) \leqslant 1$. To complete the example, we use the following fact: if $g: X \rightarrow B$ is cocyclic, then for any $\gamma \in H^{+}(X)$ and $\beta \in H^{+}(B)$, we have $\gamma \cup g^{*}(\beta)=0$. This fact follows easily from the factorization of $(g$,id) through the wedge $X \vee B$, where such cup-products are zero (cf. the inclusion $\check{G}(X ; \mathbb{Q}) \subseteq S(X ; \mathbb{Q})$ of $\left[\right.$ FLT94, Th.1]). Let $b_{m} \in H^{m}\left(S^{m}\right)$ and $b_{n} \in H^{n}\left(S^{n}\right)=H^{n}\left(S^{m} \times S^{n}\right)$ be basic classes. Then the cup-product $b_{n} \cup p_{1}^{*}\left(b_{m}\right) \neq$ $0 \in H^{m+n}\left(S^{m} \times S^{n}\right)$, so $p_{1}$ is not cocyclic.

We note that there is a third version of the category of a map, due to FadellHusseini [FH94] and studied by Cornea in [Cor98] as the relative category of a map.

Definition 3.10. Suppose given a fibration $F \stackrel{i}{\longrightarrow} E \stackrel{p}{\longrightarrow} B$. Apply the Ganea construction to obtain $F_{n}(p) \stackrel{i_{n}}{\longrightarrow} E_{n}(p) \stackrel{p_{n}}{\longrightarrow} B$ and maps $j_{n-1}: E_{n-1}(p) \rightarrow E_{n}(p)$ such that $p_{n-1}=p_{n} \circ j_{n-1}$. We define $\operatorname{cat}_{\mathrm{FH}}(p)$ to be the smallest $n$ such that $p_{n}$ has a section $s_{n}$ with $s_{n} \circ p=j_{n-1} \circ \cdots \circ j_{0}$.

Given a map $g: X \rightarrow B$, we form the induced fibration $\Omega B \longrightarrow E \stackrel{p^{\prime}}{\longrightarrow} X$ as before.

Lemma 3.11. With the above notation, we have $\operatorname{cat}(g) \leqslant \operatorname{cat}_{\mathrm{FH}}\left(p^{\prime}\right)$.

Proof. This is immediate from the definition.

Example 3.12. The inequality above will be strict whenever we find a $p^{\prime}: E \rightarrow X$ which has a section, but which is not a homotopy equivalence. For instance, let $g=*: X \rightarrow B$. Then $E=X \times \Omega B$ and $p^{\prime}$ is projection onto the first factor.

We have established a connection between a map being cocyclic and its category. Our point of view is that the category of a map is inherently of interest, and that this connection motivates interest in the notion of a cocyclic map. The dual of this connection, namely, one between the familiar notion of a cyclic map and the cocategory of a map, then serves to motivate interest in the cocategory of a map.

We summarize some results on the cocategory of a map before returning to the cocyclic/category side of things.

The fiber-cofiber construction of Definition 3.1 may be dualised in the obvious way. Starting from a cofibration $X \stackrel{j}{\longrightarrow} C \stackrel{q}{\longrightarrow} Z$, one obtains a sequence of cofibrations $X \stackrel{j_{n}}{\longrightarrow} C_{n}(j) \stackrel{q_{n}}{\longrightarrow} Z_{n}(j)$. We are interested in two cases of this construction. If we start from the cofibration $A \stackrel{j}{\longrightarrow} C A \stackrel{q}{\longrightarrow} \Sigma A$, we obtain a sequence of cofibrations dual to the Ganea fibrations. We denote these by $A \stackrel{j_{n}}{\longrightarrow} G_{n}^{\prime}(A) \stackrel{q_{n}}{\longrightarrow} F_{n}^{\prime}(A)$. If we start from the cofibration $X \stackrel{j^{\prime}}{\longrightarrow} C \longrightarrow \Sigma A$, induced via a map $f: A \rightarrow X$ from the cofibration $j: A \rightarrow C A$, then we obtain a sequence of cofibrations dual to those of (2) of Examples 3.2. We denote these cofibrations by $X \stackrel{j_{n}}{\longrightarrow} H_{n}^{\prime}(f) \stackrel{q_{n}}{\longrightarrow} K_{n}^{\prime}(f)$. Then we define $\operatorname{cocat}_{\mathrm{BG}}(f) \leqslant n$ to mean 
that there exists a map $\tilde{f}: G_{n}^{\prime}(A) \rightarrow X$ so that $\tilde{f} \circ j_{n}=f$. We define $\operatorname{cocat}(f) \leqslant n$ to mean that there exists a retraction of $j_{n}: X \rightarrow H_{n}^{\prime}(f)$. From the definitions, we have $\operatorname{cocat}_{\mathrm{BG}}(f) \leqslant \operatorname{cocat}(f)$.

Now it is easy to identify the first Ganea cofibration $A \rightarrow G_{1}^{\prime}(A)$ with the canonical map $e: A \rightarrow \Omega \Sigma A$. Furthermore, it is well-known that a space $A$ is an $H$-space if and only if this map admits a retraction. As we remarked after Definition 2.1, a cyclic map factors through the $H$-space $\operatorname{map}(X, X$; id). From this it follows that a cyclic map $f: A \rightarrow X$ factors through $\Omega \Sigma A$, and so $\operatorname{cocat}_{\mathrm{BG}}(f) \leqslant 1$.

On the other hand, we may have a map with $\operatorname{cocat}_{\mathrm{BG}}(f) \leqslant 1$ which is not cyclic.

Example 3.13. Let $f: S^{3} \rightarrow S^{3} \vee S^{3}$ be either $i_{1}$ or $i_{2}$, the inclusion into the first or second summand. Since $S^{3}$ is an $H$-space, $f$ factors through $\Omega \Sigma S^{3}$, and so cocat $_{\mathrm{BG}}(f) \leqslant 1$. But if $f$ is cyclic, then the Whitehead product $[f, \beta]=0$, for all $\beta \in \pi_{*}\left(S^{3} \vee S^{3}\right)$ [Got69, Prop.2.3]. Since $\left[i_{1}, i_{2}\right] \neq 0$, then $f$ cannot be cyclic.

The main question to be resolved is the following:

Question 3.14. For a map $f: A \rightarrow X$, is the condition that $f$ be cyclic equivalent to $\operatorname{cocat}(f) \leqslant 1$ ?

We now return to cocyclic maps and discuss a result of Halbhavi-Varadarajan in the light of the above. In [HV75, Prop.1.2], the following result is shown:

Theorem 3.15. Let $f: A \rightarrow X$ be a cyclic map. Then there is a fibration $X \rightarrow$ $E \rightarrow \Sigma A$ such that $f=\partial \circ e$, where $\partial: \Omega \Sigma A \rightarrow X$ is the connecting map of the fibration and $e: A \rightarrow \Omega \Sigma A$ is the canonical map.

This result makes clear the close relation between cyclic maps and connecting maps of fibration sequences. It is an extension of results of Gottlieb for the case in which $f: S^{n} \rightarrow X$ is a Gottlieb element. The proof makes essential use of the classifying space for fibrations with given fibre. As the authors of [HV75] point out, it is not clear if the dual of this result holds. Because there is no analogue of the classifying space in the setting of cofibrations, their proof cannot be dualized.

Theorem 3.15 implies that every cyclic map $f: A \rightarrow X$ factors through the canonical map $e: A \rightarrow \Omega \Sigma A$. We next give a result which is a slightly weaker version of the dual of Theorem 3.15, but which does establish a relation between cocyclic maps and connecting maps of cofibration sequences.

Theorem 3.16. Let $g: X \rightarrow B$ be any cocyclic map of simply connected spaces. Then there is a cofibre sequence $\Omega B \rightarrow E \rightarrow H_{1}(g)$ and a map $s: X \rightarrow H_{1}(g)$ such that $p \circ \partial \circ s=g$,

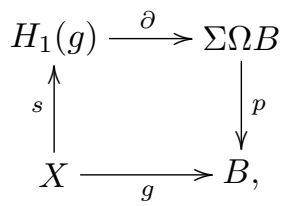

where $\partial$ is the connecting map of the cofibre sequence and $p$ is the canonical map. 
Proof. We identify $G_{1}(B) \equiv \Sigma \Omega B$ with the mapping cone of $\Omega B \rightarrow E B$ and $H_{1}(g)$ with the mapping cone of $\Omega B \rightarrow E$. By the discussion after Examples 3.2, there is a map $\tilde{g}_{1}: H_{1}(g) \rightarrow \Sigma \Omega B$ such that the following diagram commutes

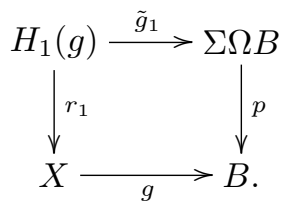

Since $g$ is cocyclic, there exists a section $s: X \rightarrow H_{1}(g)$ of $r_{1}$ by Theorem 3.7. Thus it suffices to show that $\tilde{g}_{1}=\partial: H_{1}(g) \rightarrow \Sigma \Omega B$. But this is a consequence of the third square in the following mapping of cofibre sequences

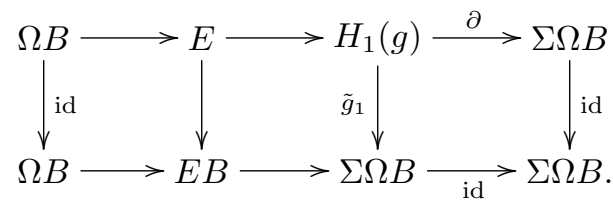

We conclude with the observation that the canonical map $p: \Sigma \Omega B \rightarrow B$ is universal for cocyclic maps into $B$.

Corollary 3.17. If $g: X \rightarrow B$ is a cocyclic map of simply connected spaces, then $g$ factors through $p: \Sigma \Omega B \rightarrow B$.

\section{References}

[ALM01] M. Arkowitz, G. Lupton, and A. Murillo, Subgroups of the group of self-homotopy equivalences, Groups of homotopy self-equivalences and related topics (Gargnano, 1999), Contemp. Math., vol. 274, Amer. Math. Soc., Providence, RI, 2001, pp. 21-32. MR 2001m:55013

[AOS05] M. Arkowitz, H. Oshima, and J. Strom, Homotopy classes of self maps and induced homomorphisms of homotopy groups, to appear, Japanese J. of Math., 2005.

[BG62] I. Berstein and T. Ganea, The category of a map and of a cohomology class, Fund. Math. 50 (1961/1962), 265-279. MR 25 \#2604

[CLOT03] O. Cornea, G. Lupton, J. Oprea, and D. Tanré, LusternikSchnirelmann category, Mathematical Surveys and Monographs, vol. 103, American Mathematical Society, Providence, RI, 2003. MR 2004e:55001 
[Cor98] O. Cornea, Some properties of the relative Lusternik-Schnirelmann category, Stable and unstable homotopy (Toronto, ON, 1996), Fields Inst. Commun., vol. 19, Amer. Math. Soc., Providence, RI, 1998, pp. 67-72. MR 99e:55003

[FH82] Y. Félix and S. Halperin, Rational LS category and its applications, Trans. Amer. Math. Soc. 273 (1982), no. 1, 1-38. MR 84h:55011

[FH94] E. Fadell and S. Husseini, Relative category, products and coproducts, Rend. Sem. Mat. Fis. Milano 64 (1994), 99-115 (1996). MR 97f:55003

[FLT94] Y. Félix, J.-M. Lemaire, and J.-C. Thomas, L'application d'évaluation, les groupes de Gottlieb duaux et les cellules terminales, J. Pure Appl. Algebra 91 (1994), no. 1-3, 143-164. MR 94j:55004

[Gan67a] T. Ganea, Induced fibrations and cofibrations, Trans. Amer. Math. Soc. 127 (1967), 442-459. MR 35 \#1025

[Gan67b] _ Lusternik-Schnirelmann category and strong category, Illinois J. Math. 11 (1967), 417-427. MR 37 \#4814

[Gan70] _ Cogroups and suspensions, Invent. Math. 9 (1969/1970), 185197. MR $42 \# 2484$

[Got69] D. H. Gottlieb, Evaluation subgroups of homotopy groups, Amer. J. Math. 91 (1969), 729-756. MR 43 \#1181

[Hil65] P. J. Hilton, Homotopy theory and duality, Gordon and Breach Science Publishers, New York, 1965. MR 33 \#6624

[HV75] I. G. Halbhavi and K. Varadarajan, Gottlieb sets and duality in homotopy theory, Canad. J. Math. 27 (1975), no. 5, 1042-1055. MR 52 \#11907

[IO03] N. Iwase and N. Oda, Decompositions of fibrations and cofibrations, to appear.

[Lim87] K. L. Lim, Cocyclic maps and coevaluation subgroups, Canad. Math. Bull. 30 (1987), no. 1, 63-71. MR 88d:55013

[LO96] G. Lupton and J. Oprea, Fixed points and powers of self-maps of $H$ spaces, Proc. Amer. Math. Soc. 124 (1996), no. 10, 3235-3239. MR 96m:55005

[LW01] K. Y. Lee and M. H. Woo, Cocyclic morphisms and dual G-sequences, Topology Appl. 116 (2001), no. 1, 123-136, Theory of fixed points and its applications (São Paulo, 1999). MR 2002f:55027

[Por71] G. J. Porter, H-fibrations, Quart. J. Math. Oxford Ser. (2) 22 (1971), 23-31. MR 44 \#5964 
[PT58] F. P. Peterson and E. Thomas, A note on non-stable cohomology operations, Bol. Soc. Mat. Mexicana (2) 3 (1958), 13-18. MR 21 \#4418

[Sig71] F. Sigrist (ed.), H-spaces. Actes de la réunion de Neuchâtel (Suisse), Août 1970, Publiés par Francois Sigrist. Textes rédigés en anglais. Lecture Notes in Mathematics, Vol. 196, Springer-Verlag, Berlin, 1971. MR 44 \#4748

[Var69] K. Varadarajan, Generalised Gottlieb groups, J. Indian Math. Soc. (N.S.) 33 (1969), 141-164 (1970). MR 43 \#6926

[Zab76] Alexander Zabrodsky, Hopf spaces, North-Holland Publishing Co., Amsterdam, 1976, North-Holland Mathematics Studies, Vol. 22, Notas de Matemática, No. 59. MR MR0440542 (55 \#13416)

This article may be accessed via WWW at http://www.rmi.acnet.ge/hha/ or by anonymous ftp at

$$
\text { ftp://ftp.rmi.acnet.ge/pub/hha/volumes/2005/n1a9/v7n1a9.(dvi,ps,pdf) }
$$

\section{Martin Arkowitz M.Arkowitz@Dartmouth.edu}

Department of Mathematics,

Dartmouth College,

Hanover NH 03755

Gregory Lupton G.Lupton@csuohio.edu

Department of Mathematics, Cleveland State University, Cleveland $\mathrm{OH} 44115$ 